\title{
Estudantes de graduação em saúde coletiva - perfil sociodemográfico e motivações
}

\author{
Undergraduate students in Public Health \\ - a socio-demographic profile and motivations
}

\author{
Marcelo Eduardo Pfeiffer Castellanos ${ }^{1}$ \\ Terezinha de Lisieux Quesado Fagundes ${ }^{1}$ \\ Tânia Celeste Matos Nunes ${ }^{2}$ \\ Célia Regina Rodrigues Gil $^{3}$ \\ Isabela Cardoso de Matos Pinto ${ }^{1}$ \\ Soraya Almeida Belisário ${ }^{4}$ \\ Solange Veloso Viana ${ }^{1}$ \\ Guilherme Torres Correa ${ }^{5}$ \\ Raphael Augusto Teixeira de Aguiar ${ }^{4}$
}

\footnotetext{
${ }^{1}$ Instituto de Saúde Coletiva, Universidade Federal da Bahia. Rua Basílio da Gama s/n, Canela. 40110-040 Salvador BA. mcastellanos73@gmail.com ${ }^{2}$ Escola Nacional de Saúde Pública, Fiocruz.

${ }^{3}$ Departamento de Saúde Coletiva, Centro de Ciências da Saúde, Universidade Estadual de Londrina.

${ }^{4}$ Faculdade de Medicina, UFMG.

${ }^{5}$ Faculdade de Educação,

USP.
}

\begin{abstract}
Undergraduate Courses in Public Health (UCPH) feature a new way of teaching in the Public Health field that aims to educate professionals focused on the main health needs of the Brazilian population and on the Unified Health System. Thus, it is relevant to know the students' socio-demographic profile including their interests in relation to education and professional development. Adopting a quantitative approach with these objectives, a survey was conducted with 304 UCPH students who filled out a semi-structured questionnaire in 2010 in the following federal universities: Acre, Bahia, Minas Gerais, Mato Grosso, Paraná, Rio de Janeiro and Rio Grande do Norte. The overwhelming majority was female, around 26 years of age, single, living with their parents, mostly attended public school, came from lower classes with lower educational background, approved night classes, worked, and contributed to their family incomes. They aspired to work in public sector - preferably in the state capitals and they saw UCPH as an opportunity to work in the public health field. This profile reveals challenges and advances in the intended education, reinforcing the need to monitor the UCPH development process.
\end{abstract}

Key words Students, Career choice, Motivation, Public Health studies, Education in Public Health
Resumo Os Cursos de Graduação em Saúde Coletiva (CGSC) consistem em uma nova modalidade formativa no campo da Saúde Coletiva que visa formar profissionais focados nas principais necessidades em saúde da população brasileira e no Sistema Único de Saúde. Assim, torna-se relevante traçar o perfil sociodemográfico de seus alunos e conhecer seus interesses quanto à formação e atuação profissional. Com esses objetivos, em 2010, adotando uma abordagem quantitativa ao tema, realizamos um inquérito, através da aplicação de um questionário semiestruturado aos alunos dos CGSC de universidades federais dos estados do Acre, Bahia, Minas Gerais, Mato Grosso, Paraná, Rio de Janeiro e Rio Grande do Norte. Entre os 304 respondentes, encontramos uma população majoritariamente feminina, com 26 anos de idade, composta por indivíduos solteiros que moram com os pais, cursaram principalmente o ensino público, pertencem a famílias com baixa renda e escolaridade, aprovam o estudo noturno, trabalham e contribuem com a renda familiar, desejam trabalhar no setor público, preferencialmente em capitais e identificam no CGSC uma oportunidade para trabalhar na área da saúde. Esse perfil aponta para desafios e avanços presentes na formação pretendida, reforçando a necessidade de monitoramento do processo de implementação dos CGSC.

Palavras-chave Estudantes, Escolha da profissão, Motivação, Estudos em saúde coletiva, Educação e Saúde Pública 


\section{Introdução}

O processo de implantação de Cursos de Graduação em Saúde Coletiva (CGSC) no Brasil avança rapidamente, iniciando um novo capítulo no debate sobre esta modalidade formativa. Vale lembrar que este debate não é novo, tendo se iniciado há cerca de duas décadas e se estruturou mais sistematicamente, nos meios acadêmicos, com uma série de oficinas, mesas redondas e encontros dedicados ao tema, realizados nos últimos dez anos ${ }^{1}$. O Programa de Apoio a Planos de Reestruturação e Expansão das Universidades Federais (Reuni), implementado através do Decreto $n^{\circ} 6.096 / 2007^{2}$, impulsionou esse processo nas universidades públicas federais. Nos dois anos seguintes à publicação desse Decreto, foram abertos cerca de dez cursos em instituições de ensino federal (IFES) no Brasil.

Ainda que a graduação em Saúde Pública não seja uma experiência nova no contexto internacional ${ }^{1,3,4}$, ela ganha especificidades quando projetada no campo da Saúde Coletiva (SC) brasileira. Diversos são os motivos que justificaram a implantação dessa modalidade formativa no país ${ }^{1,5,6}$, dentre os quais, destacam-se três para fins deste artigo.

Em primeiro lugar, os CGSC procuram responder ao aumento da demanda por profissionais com formação específica em Saúde Coletiva para atuar no Sistema Único de Saúde (SUS), em vista de sua expansão. Os CGSC responderiam a essa demanda com maior agilidade e magnitude do que a pós-graduação, já que esta última pressupõe um itinerário escolar mais longo para formar um profissional de Saúde Coletiva. Também, responderiam de maneira mais efetiva do que a oferta de disciplinas de Saúde Coletiva, geralmente, marginalizada nas graduações da área da saúde; e de maneira muito mais robusta do que os cursos de especialização.

Em segundo, a Graduação em Saúde Coletiva reflete o acúmulo de experiências de ensino em graduação e pós-graduação no país, representando a consolidação do processo de institucionalização das práticas de ensino da Saúde Coletiva e avanço decisivo em direção a uma formação interdisciplinar em nível de graduação. Os CGSC evitariam, assim, a "reprofissionalização" ocorrida na pós-graduação, quando esta recebe profissionais com uma formação disciplinar, geralmente orientada pelo modelo biomédico. Eles implicam, também, um novo e forte impulso na consolidação e expansão de uma carreira profissional específica na área.
Em terceiro, espera-se que a abertura dos cursos represente uma nova força mobilizadora do processo de Reforma Sanitária Brasileira (RSB), a partir da formação de um novo ator social no campo da Saúde Coletiva, comprometido com os princípios e valores éticos e políticos que inspiram tal Reforma. Esse ator se conforma tanto pelos alunos e egressos dos CGSC, que já encontram suas formas de organização e representação estudantil (local e nacional), com destaque para a Coordenação Nacional dos Estudantes de Saúde Coletiva (CONESC). Mas também, pelas próprias instituições que sediam os CGSC, as quais já encontraram sua forma de articulação política através do Fórum de Graduação em Saúde Coletiva. Este está abrigado na Associação Brasileira de Saúde Coletiva (ABRAS$\mathrm{CO}$ ), o que representa um importante passo em direção ao compromisso com os princípios, as dinâmicas, os valores e os compromissos da Saúde Coletiva, diminuindo assim o risco de alguns cursos ficarem subsumidos a questões e processos internos ao contexto universitário.

Essas justificativas apontam para especificidades do caso brasileiro e levantam algumas questões, tais como: Qual(is) o(s) perfil(is) profissional(is) demandado(s) pelo SUS, no que se refere à Saúde Coletiva? Quais são as capacidades e interesses das instituições de ensino de nível superior para formar profissionais com esse(s) perfil(is)? Os interesses que mobilizam os alunos a ingressarem nos CGSC se harmonizam com as justificativas e metas que orientam tais cursos? O perfil sociodemográfico dos alunos traz implicações para o tipo de formação pretendida nos cursos?

Entendemos que o SUS necessita de um profissional com formação interdisciplinar, obtida através de sólida incorporação dos saberes da Saúde Coletiva, que o qualifique como um ator estratégico apto a atuar em um sistema de saúde que adquire caráter cada vez mais extenso, complexo e especializado - portanto, desafiador em termos de políticas sociais comprometidas com a participação e o bem estar social ${ }^{5,7-9}$.

As instituições de ensino superior públicas parecem se mostrar interessadas nos CGSC, uma vez que a abertura desses cursos ocorreu em instituições federais e estaduais de ensino, em todas as regiões do país.

Torna-se relevante e urgente traçar o perfil sociodemográfico dos alunos matriculados nos CGSC, bem como conhecer seus interesses quanto à formação e atuação profissional, o que nos dirige às duas últimas questões acima apresentadas. 
Estudos sobre o perfil dos alunos no campo da saúde têm levado à caracterização da sua clientela ${ }^{10}$ e de suas evoluções e mudanças ${ }^{11}$, com destaque para a comparação dos perfis socioeconômicos $^{12}$, procurando explorar questões vocacionais ${ }^{13}$, de mercado de trabalho ${ }^{14}$ e de ensinoaprendizagem $^{15}$. Porém, dado o caráter recente da abertura dos CGSC, ainda não existem estudos sobre o perfil de seus alunos.

A presente investigação objetivou caracterizar, preliminarmente, o perfil dos alunos ingressantes em CGSC ofertados em IFES das cinco regiões do Brasil. Esse perfil incidiu sobre características sociodemográficas, interesses e expectativas em relação à formação e exercício profissional em Saúde Coletiva. Os dados apresentados neste artigo foram produzidos no âmbito do projeto "Análise da Implantação dos Cursos de Graduação em Saúde Coletiva em Diferentes Regiões do Brasil”, desenvolvido pela Associação Brasileira de Saúde Coletiva (ABRASCO), através do seu Grupo Temático (GT) - Trabalho e Educação na Saúde. Esse projeto contou com o apoio da Organização Pan-Americana da Saúde (OPAS) e da Secretaria de Gestão do Trabalho e da Educação na Saúde do Ministério da Saúde (SGTES/MS).

\section{Metodologia}

Trata-se de uma investigação exploratória, com emprego de abordagem metodológica quantitativa, baseada em dados primários obtidos através da aplicação de um questionário semiestruturado aos alunos matriculados no primeiro semestre de 2010, nos cursos de graduação em Saúde Coletiva implantados nas universidades federais dos estados do Acre (UFAC), Bahia (UFBA), Minas Gerais (UFMG), Mato Grosso (UFMT), Paraná (UFPR), Rio de Janeiro (UFRJ) e Rio Grande do Norte (UFRN). Procuramos incluir, no presente inquérito, todos os cursos em instituições de ensino superior (IES) públicas, abertos até o momento da aplicação do questionário. Porém, por razões internas da Universidade Federal do Rio Grande do Sul, e, em vista de uma greve de estudantes na Universidade de Brasília não foi possível aplicar o questionário nessas IES.

Os cursos investigados possuem as seguintes denominações: Graduação em Saúde Coletiva (UFMT, UFBA, UFRJ, UFAC, UFPR), Análise de Sistemas e Serviços de Saúde (UFMG), Gestão em Sistemas e Serviços de Saúde (URFN).

O questionário, elaborado pela equipe de investigadores, foi pré-testado em uma turma de estudantes do CGSC do Instituto de Saúde Coletiva da Universidade Federal da Bahia (ISC/ UFBA) e revisado por três pesquisadores desse Instituto, externos à equipe de pesquisa.

Apresentaremos os resultados de dois blocos de questões: 1. Características sociodemográficas dos estudantes (faixa etária, sexo, estado civil, vínculo de moradia, renda média mensal da família, atividade remunerada atual, participação na renda familiar, trabalho remunerado e área de atuação, experiência de trabalho no SUS, percurso escolar e escolaridade dos pais); 2. Opção pelo curso e expectativas em relação ao curso e ao exercício profissional.

Os questionários foram aplicados pelos membros da equipe de pesquisa nas dependências das IES selecionadas. Todos os respondentes assinaram o Termo de Consentimento Livre e Esclarecido, antes de participar da pesquisa. A qual foi aprovada pelo Comitê de Ética em Pesquisa do ISC/UFBA.

Procurou-se aplicar o questionário a todos os alunos dos cursos selecionados, envolvendo assim estudantes do primeiro ao quarto período curricular (semestre). Por razões locais diversas, essa meta foi atingida em graus variáveis, tendo sido entrevistados todos os 38 alunos da UFBA (100\%), 70 dos 78 alunos da UFRN (90\%), 95 dos 128 da UFMG (74\%), 21 dos 34 da UFMT (62\%), 20 dos 34 da UFRJ (59\%), 15 dos 19 da UFPR (79\%) e 45 dos 80 da UFAC (56\%).

O total de 304 respondentes encontrava-se distribuído nos seguintes períodos curriculares: 148 do primeiro período (49\%); 67 do segundo (22\%); 66 do terceiro $(21,4 \%) ; 23$ do quarto (7,6\%). E distribuíram-se nos cursos, da seguinte maneira: 95 da UFMG $(31,3 \%) ; 70$ da UFRN $(23,0 \%) ; 38$ da UFBA $(12,5 \%) ; 45$ da UFAC (14,8\%); 20 da UFMT (6,9\%); 21 da UFRJ $(6,6 \%)$ e 15 da UFPR (4,9\%).

A tabulação de dados e sua revisão foram realizadas por auxiliares de pesquisa, acompanhados por um dos pesquisadores. A categorização e a análise das respostas ficaram a cargo dos próprios pesquisadores. Todos os procedimentos foram verificados e discutidos por diferentes membros da equipe de pesquisa. Para os dados das perguntas fechadas foram calculadas frequências absolutas e percentuais, através do uso do programa EPIINFO. Classificamos as respostas relativas à motivação para entrada no curso através de categorias de Soares ${ }^{16}$ e Bardagi et al. ${ }^{17}$, adaptadas para este estudo, conforme se segue: motivos de ordem profissional, de ordem socioeconômica e de ordem pessoal. 
Os projetos político-pedagógicos (PPP) dos cursos selecionados foram consultados como uma fonte específica de dados sobre os objetivos e perfis do egresso dos CGSC, cujas principais tendências e ênfases foram sintetizadas para permitir comparações com o perfil delineado através do questionário, ampliando assim a análise e a discussão dos seus resultados.

\section{Resultados}

Sinteticamente, o perfil dos alunos dos CGSC é caracterizado por uma população com 26 anos de idade em média, majoritariamente: feminina, solteira, que mora com os pais, cursou o ensino público, integra famílias com baixa renda e baixa escolaridade, aprova o estudo noturno, trabalha e contribui com a renda familiar, deseja trabalhar no setor público, preferencialmente em capitais, e vê nos CGSC uma oportunidade para trabalhar na área da saúde.

\section{Características sociodemográficas}

A participação feminina crescente nos cursos da área de saúde reflete-se também nos CGSC. Aproximadamente dois terços $(78,6 \%)$ dos respondentes era do sexo feminino (Tabela 1 ).

Tabela 1. Características demográficas dos estudantes dos cursos de graduação em Saúde Coletiva, em universidades selecionadas. Brasil, 2010. $(\mathrm{N}=304)$.

\begin{tabular}{lrr}
\hline \multicolumn{1}{c}{ Características demográficas } & N & $\%$ \\
\hline Sexo & 65 & 21,4 \\
$\quad$ Masculino & 239 & 78,6 \\
$\quad$ Feminino & & \\
Faixa etária & 82 & 27,6 \\
$\quad$ < 20 anos & 155 & 50,4 \\
20 a 29 anos & 40 & 13,4 \\
30 a 39 anos & 17 & 5,6 \\
40 a 49 anos & 05 & 1,4 \\
50 a 59 anos & 01 & 0,3 \\
Igual ou maior de 60 anos & 04 & 1,3 \\
$\quad$ SR & & \\
Estado civil & 236 & 78,0 \\
$\quad$ Solteiro (a) & 44 & 14,0 \\
$\quad$ Casado (a) & 12 & 4,0 \\
$\quad$ Vive com companheiro (a) & 09 & 3,0 \\
$\quad$ Divorciado (a)/separado (a) & 03 & 1,0 \\
$\quad$ Viúvo (a) & & \\
\hline
\end{tabular}

$\mathrm{SR}=$ sem resposta
A idade dos respondentes variou entre 17 e 68 de idade, com uma concentração entre 17 e 29 de idade $(78,0 \%)$, sendo que $50,4 \%$ declararam ter entre 20 e 29 anos de idade (Tabela 1). Vale destacar que $20,7 \%$ possuíam 30 ou mais anos de idade.

Quanto ao estado civil, os dados da Tabela 1 demonstram que $70,0 \%$ dos estudantes se declararam solteiros, $14,5 \%$ casados, $3,9 \%$ viviam com companheiros, $2,6 \%$ eram divorciados e $1,0 \%$ viúvos, respectivamente.

O perfil demográfico encontrado é de uma população majoritariamente feminina, de jovens com até 29 anos de idade e solteiros.

Quanto à escolaridade, tomando por referência o nível fundamental, 51,3\% dos alunos realizaram esse nível exclusivamente em escolas públicas, $36,9 \%$ em escolas privadas e 11,8\% em ambas (Tabela 2).

Cerca de um quarto das mães e um terço dos pais dos respondentes possuem apenas o ensino fundamental completo. Cerca de um quarto de ambos possuem o nível médio completo. Quando somados todos que iniciaram o fundamental e/ou médio, tendo completado ou não esses níveis, encontramos um pouco mais de $60 \%$ dos pais e mães. Em torno de um quarto dos pais e um terço das mães tiveram acesso ao nível superior, mesmo que incompletos (Tabela 2).

Mais da metade do alunado respondente $(68,7 \%)$ pertencem às famílias cuja renda não ultrapassa 6 salários mínimos (SM), sendo que, mais de um quarto atinge apenas $3 \mathrm{SM}$ e apenas $9,9 \%$ possuem renda familiar igual ou superior a 11 SM (Tabela 2).

Mais de $60 \%$ dos respondentes moram com pais e irmãos; $53,3 \%$ trabalham e 33,6\% participam na renda familiar. Do total, 17,4\% moravam com o cônjuge/companheiro (a) e 20,1\% em outras situações (Tabela 2).

Conforme Tabela 3, a maioria (74\%) dos respondentes disse não ter experiência com o SUS, enquanto $23 \%$ declararam ter alguma experiência anterior ao ingresso no curso. Do total de respondentes, $14,1 \%$ já tinham completado outro curso de graduação no momento do ingresso no CGSC e 11,5\% estavam frequentando simultaneamente outro curso de graduação. Cerca de um quarto dos alunos direciona-se para uma dupla diplomação em graduação. Mais de 60\% dos estudantes respondentes frequentavam CGSC no turno noturno, $30,5 \%$ no turno diurno.

A maioria dos estudantes (61,9\%) disse que, após o término do CGSC, gostaria de trabalhar no setor público, $6,9 \%$ no setor privado e $25,7 \%$ não indicou preferência (Gráfico 1). Quase me- 
Tabela 2. Características socioeconômicas dos estudantes dos cursos de graduação em Saúde Coletiva e das famílias de origem, em universidades selecionadas. Brasil, 2010. $(\mathrm{N}=304)$.

\begin{tabular}{ccc}
\hline Indicador socioeconômico & $\mathrm{N}$ & $\%$ \\
\hline
\end{tabular}

\begin{tabular}{lrr}
\hline $\begin{array}{l}\text { Dependência administrativa do curso } \\
\text { realizado no nível fundamental }\end{array}$ & \\
$\quad$ Escola pública & 156 & 51,3 \\
Escola privada & 112 & 36,9 \\
Escola pública e privada & 36 & 11,8 \\
SR & - &
\end{tabular}

Renda mensal da família de origem (em salário mínimo)

$<$

4 a 6

7 a 10

11 a 15

Maior ou igual a 16

SR

Coabitação dos estudantes

Com a família

Com cônjuge/companheiro/a

Com outros parentes

Sozinho/a

Com amigos e/ou colegas

Em pensionato

Em residência universitária

Outros

SR

Tem atividade remunerada atual

Sim

Não

Às vezes

SR

Participa na renda familiar

Sim

Não

Às vezes

SR

Nível de escolaridade dos pais

Pai

Fundamental incompleto

Fundamental completo

Médio incompleto

Médio completo

Superior incompleto

Superior completo

SR

Mãe

Fundamental incompleto

Fundamental completo

Médio incompleto

Médio completo

Superior incompleto

Superior completo

SR

$80 \quad 26,3$

12942,4

$58 \quad 19,1$

258,2

051,7

$07 \quad 2,3$

$190 \quad 62,5$

$53 \quad 17,4$

$24 \quad 7,9$

$14 \quad 4,6$

$10 \quad 3,3$

$01 \quad 0,3$

$04 \quad 1,3$

$06 \quad 2,0$

$02 \quad 0,7$

16253,3

12841,8

103,3

$04 \quad 1,6$

10133,6

15350,3

4915,8

010,3

\begin{tabular}{rr}
91 & 29,9 \\
15 & 4,9 \\
32 & 10,5 \\
77 & 25,3 \\
13 & 4,3 \\
63 & 20,7 \\
13 & 4,3 \\
& \\
75 & 24,6 \\
16 & 5,2 \\
24 & 7,9 \\
82 & 27,0 \\
18 & 6,0 \\
80 & 26,3 \\
09 & 3,0 \\
\hline
\end{tabular}

$\mathrm{SR}=$ sem resposta tade $(48,7 \%)$ tem preferência em trabalhar na capital dos seus estados, $13,1 \%$ no interior e uma parcela significativa $(38,2 \%)$ declarou não ter preferência por localidade específica (Gráfico 2).

Tabela 3. Características formativas e laborais dos estudantes dos cursos de graduação em Saúde Coletiva, em relação à Saúde Coletiva, em universidades selecionadas. Brasil, 2010. $(\mathrm{N}=304)$.

\begin{tabular}{|c|c|c|}
\hline $\begin{array}{c}\text { Características em relação } \\
\text { à Saúde Coletiva }\end{array}$ & $\mathbf{N}$ & $\%$ \\
\hline \multicolumn{3}{|l|}{ Experiência de trabalho no SUS } \\
\hline Sim & 70 & 23,0 \\
\hline Não & 227 & 74,7 \\
\hline SR & 07 & 2,3 \\
\hline \multicolumn{3}{|l|}{ Completou outro curso de graduação } \\
\hline Sim & 43 & 14,1 \\
\hline Não & 201 & 66,2 \\
\hline Começou mas não terminou & 60 & 19,7 \\
\hline SR & - & - \\
\hline \multicolumn{3}{|l|}{ Aluno regular de outra graduação } \\
\hline Sim & 35 & 11,5 \\
\hline Não & 259 & 85,2 \\
\hline SR & 10 & 3,3 \\
\hline \multicolumn{3}{|l|}{ Turno de frequência no curso } \\
\hline Diurno & 93 & 30,5 \\
\hline Noturno & 186 & 61,2 \\
\hline Diurno e noturno & 04 & 1,3 \\
\hline SR & 21 & 7,0 \\
\hline
\end{tabular}

$\mathrm{SR}=$ sem resposta

Brasil, $2010(\mathrm{~N}=304)$

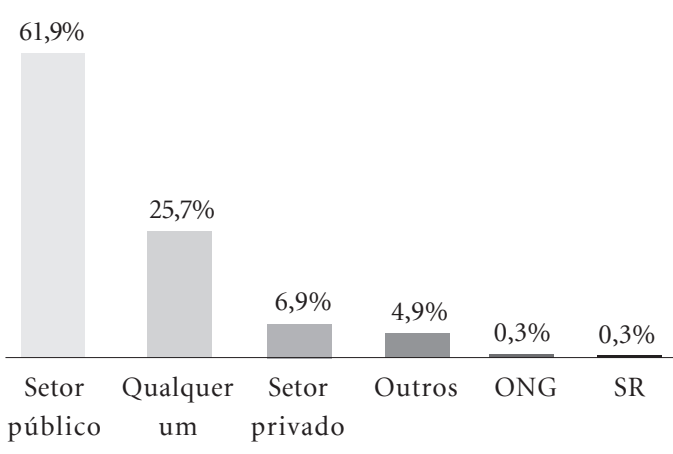

Gráfico 1. Setor preferencial para ingresso profissional apontado pelos estudantes dos cursos de graduação em Saúde Coletiva, em selecionadas universidades. 


\section{Motivações}

Identificamos que, dentre as motivações para a escolha do CGSC, cerca de $45,7 \%$ são de ordem profissional, 35,2\% de ordem pessoal e $14,8 \%$ de ordem socioeconômica (Tabela 4).

Dentre as motivações profissionais, "trabalhar na área da saúde” destacou-se em relação às demais respostas, ficando reforçada pelas duas respostas seguintes, que apontam para inserção profissional na área da saúde, com uma formação específica ou complementar em Saúde Coletiva. A motivação em contribuir para a Saúde Coletiva/Pública e com o SUS foi mencionada por um terço das respostas classificadas nesta categoria.

Dentre as motivações pessoais, a maior parte dos respondentes apontou gostar e/ou sentir afinidade com a área da saúde. Notou-se também interesse pelo caráter inaugural do curso no plano das graduações da área da saúde, por ser um curso "novo/inovador" presente em cerca de 10\% das respostas presentes nesta categoria. Porém, cerca de $20 \%$ das respostas abrangeram "curiosidade/interesse" ou "segunda opção do vestibular", não se consubstanciando, talvez, em um forte interesse específico.

Dentre as motivações socioeconômicas, destacou-se ter expectativa positiva em relação à entrada no mercado de trabalho.

Ao consultarmos os Projetos Político-Pedagógicos dos cursos incluídos neste estudo, foram

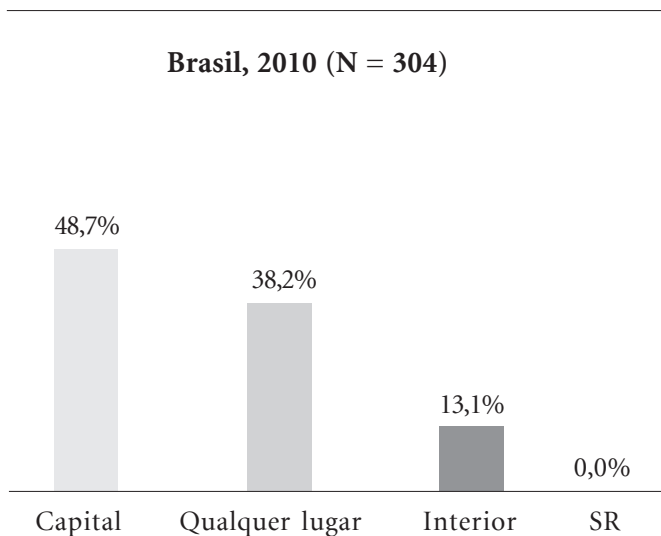

Gráfico 2. Localidade preferencial para ingresso profissional apontado pelos estudantes dos cursos de graduação em Saúde Coletiva, em selecionadas universidades. identificados dois enfoques principais dados aos objetivos e perfis de egressos. De um lado, os PPP dos cursos da UFMG e da UFRN mantém um enfoque específico em gestão (ainda que aquele último faça menção a um perfil generalista), de forma coerente com suas nomenclaturas de curso. De outro lado, todos os outros cursos enfocam a Saúde Coletiva, de modo claro e abrangente, descrevendo saberes e áreas de atuação no perfil de seus egressos e objetivos do curso.

Tomando por referência esse último enfoque, majoritário nos PPP consultados, produzimos uma síntese em que o perfil do egresso aponta para um profissional com sólida formação interdisciplinar que o habilita a realizar análises e intervenções em saúde que levam em consideração seus contextos social, político e econômico. De modo mais específico, essas análises e intervenções incidem sobre as condições, situações e práticas de saúde, tendo-se em perspectiva o processo saúde-doença-cuidado, os processos de trabalho em saúde, os modelos de atenção e o

Tabela 4. Motivações dos alunos para a escolha do CGSC, em cursos selecionados. Brasil, 2010 $(\mathrm{N}=304)$

\begin{tabular}{lrr}
\hline \multicolumn{1}{c}{ Motivações } & N & \multicolumn{1}{c}{$\%$} \\
\hline $\begin{array}{lrr}\text { Motivos profissionais } \\
\text { Trabalhar na área/gestão da saúde }\end{array}$ & 42 & 13,82 \\
$\quad$ Inserção anterior na área da saúde & 31 & 10,20 \\
$\quad$ trabalho, estudo) & & \\
Ampliar/complementar & 23 & 7,57 \\
conhecimentos & & \\
Contribuir para Saúde Coletiva e SUS & 35 & 11,51 \\
$\quad$ Atuação multidisciplinar e /ou & 08 & 2,63 \\
integrada com social & & \\
Motivos socioeconômicos & & \\
$\quad$ Potencialidades do mercado de & 45 & 14,80 \\
trabalho & & \\
Motivos pessoais & & \\
$\quad$ Gostar/afinidade com área da Saúde & 54 & 17,76 \\
$\quad$ Segunda opção no vestibular & 15 & 4,93 \\
Interesse/curiosidade & 10 & 3,29 \\
Curso novo/inovador & 10 & 3,29 \\
$\quad$ Realização pessoal & 07 & 2,30 \\
Curso noturno & 04 & 1,32 \\
Influência rede social & 04 & 1,32 \\
Falta de opção e facilidade para entrar & 03 & 0,99 \\
Outros & $\mathbf{0 2}$ & $\mathbf{0 , 6 6}$ \\
SR & $\mathbf{1 1}$ & $\mathbf{3 , 6 2}$ \\
Total & $\mathbf{3 0 4}$ & $\mathbf{1 0 0}$ \\
\hline SR = sem resposta & & \\
& &
\end{tabular}


Por um lado, as expectativas dos alunos em relação à atuação profissional, centradas no setor público, são convergentes com o compromisso dos cursos em formar profissionais de Saúde Coletiva prioritariamente para o SUS. Essa expectativa pode refletir a percepção sobre a expansão do mercado de trabalho em saúde no setor público, impulsionada pelo SUS, onde predominam profissionais de nível superior ${ }^{18}$; a sensibilização para a atuação no SUS realizada nas atividades do curso; ou ainda a expectativa favorável dos alunos em torno da abertura de concursos públicos para os egressos dos cursos.

Por outro lado, o perfil dos ingressantes nos CGSC e as razões que motivaram tal ingresso apontam para sérios desafios postos em relação aos objetivos e justificativas dos cursos. Nesse sentido, se os respondentes, em sua maioria, desejam trabalhar no setor público de saúde, poucos têm contato anterior com o SUS. Além disso, o fato da maior parte realizar o curso à noite e trabalhar durante o dia - muitas vezes, contribuindo com a renda familiar - aponta para o grande desafio enfrentado na inserção desses alunos em

campos de prática e de estágio, ao longo de sua formação. Essa característica também aponta para uma sobrecarga de trabalho e menor tempo para o estudo presente entre os alunos.

Soma-se a isso o fato de somente uma minoria dos respondentes (11\%) ter ingressado no CGSC motivada por claro interesse em contribuir para a Saúde Coletiva e para o SUS. E uma parcela menor ainda (3\%) ingressou motivada pelo desejo de uma atuação multidiciplinar ou integrada com o social. A análise geral das motivações sugere um interesse pragmático voltado à inserção no mercado de trabalho em saúde. Uma parcela considerável dos alunos $(25,6 \%)$ possui ou realiza outra graduação, o que pode indicar a ideia de que a Saúde Coletiva represente para eles uma complementação dos estudos, em uma área "especializada", como forma de viabilizar a entrada em um nicho de mercado.

Ainda que essa situação possa refletir um momento inaugural de uma formação ainda pouco conhecida ao nível de graduação, tais resultados devem ser levados em consideração pelos gestores dos CGSC. Percebemos a importância dessa consideração quando lembramos - em conformidade com os PPP consultados - que os CGSC pretendem formar um profissional com formação interdisciplinar focado em análises de situação e intervenções que considerem aspectos sociais, políticos e econômicos. Portanto, um profissional com perfil desafiador para os processos formativos tradicionalmente existentes na

Quadro 1. Síntese do perfil do egresso do CGSC.

GERAL:

Profissional de saúde humanista, com formação interdisciplinar, empreendedor, crítico, com visão ampla do contexto social, político e econômico das situações e intervenções em saúde.

\section{ESPECÍFICO:}

Apto para a compreensão e atuação em de todos os níveis de Gestão, Vigilância e Atenção em saúde, com foco na organização, planejamento e avaliação dos processos institucionais, incluindo-se ações voltadas ao sistema de saúde e aos processos de trabalho, pautadas pela transversalidade dos saberes;

Capaz de analisar diferentes situações de saúde, identificando suas dimensões sociais, políticas, econômicas e culturais, em especial, a partir dos aportes das Ciências Humanas e Sociais;

Capaz de identificar e analisar os interesses e perspectivas dos sujeitos do processo saúde-doença-cuidado, de forma a atuar como mediador, articulador e líder na pactuação de ações e intervenções dos serviços, sistemas e políticas de saúde negociadas entre esses sujeitos em prol de metas voltadas à promoção, proteção e recuperação da saúde e da qualidade de vida, observando-se, assim, princípios éticos de respeito à vida, à diversidade cultural e à participação social.

Fonte: Projetos Político-Pedagógicos e/ou sites dos cursos selecionados (UFBA, UFRJ, UFAC, UFPR, UFMT). 
área da saúde, que também ganha relevância ao pretender uma relação estreita com o mundo do trabalho no SUS. Devemos levar em consideração que a expansão do SUS - associada, principalmente, com o avanço do processo de descentralização e de regionalização - tem aumentado o espectro e a complexidade das ações e pactuações realizadas, especialmente, nos níveis estadual e municipal de saúde. Esse processo de expansão e consolidação do SUS implica o reordenamento das funções regulatórias, gerenciais e de prestação de serviços ${ }^{8,19,20}$.

Diversos estudos têm apontado para uma rica e complexa relação entre motivações para o ingresso em curso superior e expectativas profissionais, que procuraram analisar os resultados de quatro grandes inquéritos sociológicos que forneceram importantes dados para identificar em que medida as escolhas ao longo da trajetória acadêmica dos diplomados em nível superior, em Portugal, são influenciadas por razões ligadas à sua (futura) atividade profissional ${ }^{21}$. Se, por um lado, os diplomados informaram buscar o nível superior como forma de entrada qualificada em um mercado de trabalho cada vez mais marcado pela insegurança e precariedade dos contratos; por outro lado, eles também demonstraram estar fortemente guiados por outras motivações tais como "gosto ou interesse pelo estudo" ou com o "aprofundamento de conhecimentos" ou com o "desenvolvimento intelectual". Desse modo, a escolha esteve orientada tanto por questões contextuais pragmáticas, quanto por valores mais amplos de reafirmação do ensino superior como fonte de saber.

Ao investigarem as razões para o ingresso nos cursos de enfermagem, fisioterapia e nutrição, na Pontifícia Universidade Católica do Rio Grande do Sul (PUCRS), pesquisadores encontraram um arranjo de motivações formuladas no contexto familiar, nas representações sociais sobre as profissões e nas expectativas favoráveis sobre a entrada no mercado de trabalho ${ }^{22}$. Os alunos demonstraram sofrer forte influência familiar (especialmente de parentes que já exerciam a profissão) e das imagens sociais sobre a profissão. De maneira semelhante, a escolha pelo curso de enfermagem entre alunos de uma escola pública e outra privada, ambas do Rio de Janeiro, esteve influenciada pelo interesse na área de saúde (ainda que enfermagem não apareça como primeira opção), por gostar de cuidar de pessoas, pela identificação de maiores chances de ingresso em um curso superior e de um mercado de trabalho promissor $^{23}$. Dentre as expectativas futuras, desta- caram-se realização profissional e pessoal, retorno financeiro e carreira. Portanto, aqui também há uma composição de pragmatismo e ideais pessoais e/ou sociais.

O perfil socioeconômico dos respondentes e de seus pais reforça a ideia de que o ingresso no ensino superior e a escolha do CGSC se relacionam a um interesse pragmático voltado à inserção no mercado de trabalho. Também, representa mais um desafio à perspectiva interdisciplinar pretendida pelos cursos, uma vez que esta se beneficiaria de um contato o mais intenso e ampliado possível com bens e serviços culturais, assim como de uma sólida formação escolar.

Notamos na literatura que o perfil dos respondentes se aproxima daquele encontrado entre os alunos de enfermagem e se distancia do perfil dos alunos de farmácia, odontologia e terapia ocupacional, no que se refere a idade, gênero, escolarização (própria e dos pais), renda individual e familiar e taxa ocupacional ${ }^{24}$.

O fato da oferta dos cursos ocorrer em período noturno em mais de $80 \%$ e de cerca de $60 \%$ dos respondentes aprovar esse turno para seus estudos, juntamente com as situações acima descritas, reforçam a ideia de que a abertura dos CGSC, impulsionada pelo Reuni, insere-se em um contexto de ampliação do acesso ao ensino superior e de inclusão de segmentos populacionais tradicionalmente excluídos desse nível de escolaridade. Devemos lembrar que apenas um quarto dos pais e um terço das mães desses alunos tiveram algum tipo de acesso ao nível superior. A qualificação escolar e profissional desses alunos poderá, nesse contexto, contribuir para a redução das inequidades do ensino superior brasileiro.

Se considerarmos que a inclusão social é um dos valores significativos ao campo da Saúde Coletiva, manter e fortalecer uma inclusão que garanta a qualificação formativa e profissional de seus futuros egressos é um dos grandes desafios atuais dos cursos. Desafio esse que requisitará respostas em diferentes contextos e direções, envolvendo reflexões e ações em relação às estruturas curriculares e processos de ensino-aprendizagem; aprimoramento da formação em práticas pedagógicas e da experiência docente no CGSC; amadurecimento de currículos e processos pedagógicos com foco na saúde coletiva e nas necessidades contemporâneas do SUS; apoio à inserção ampliada do alunado no contexto da vida universitária e acadêmica (envolvimento em atividades de pesquisa e extensão, preferencialmente apoiadas por bolsas, na organização estudantil), dentre outros elementos. 
Vale lembrar que, entre aqueles que informaram exercer atividade remunerada, praticamente metade o faz na área da saúde. Assim, se essa situação ameaça a qualificação dos processos de ensino-aprendizagem, vivenciados por esses alunos, ela também pode se constituir em oportunidade no contexto das atividades formativas, gerando reflexões as experiências e vivências no mundo do trabalho, relevantes para o conjunto dos alunos do curso.

Ainda que os alunos tenham uma clara preferência por trabalhar nas capitais, há aqueles abertos a trabalhar em outras localidades. Assim, pouco mais de $13 \%$ dos respondentes declararam preferência pelo interior, ao passo que quase $25 \%$ identificaram a existência de melhores oportunidades de trabalho nessas localidades. Esse interesse discente pela atuação profissional no interior do estado pode ser explorado e ampliado pelos docentes e coordenadores, uma vez que a avassaladora maioria dos municípios do país se situa no interior, onde se espera uma elevada demanda por profissionais de saúde altamente qualificados em Saúde Coletiva.

Vale lembrar que o "processo de escolha profissional não se encerra com o ingresso no curso escolhido. No início de sua trajetória acadêmica, o estudante reavalia a sua opção frente às experiências, vivências e conhecimento acerca da profissão, podendo reafirmar ou questionar sua escolha (...). Tanto a escolha profissional como as vivências acadêmicas dos participantes ocorrem no contexto dos conflitos de poder das profissões da saúde"22. Trata-se de uma observação importante, pois aponta para o desafio aberto aos gestores dos CGSC no que se refere, de um lado, à mobilização dos alunos em torno dos enfoques, objetivos e justificativas dos cursos, e de outro lado à aproximação e diálogo com as perspectivas, desejos e interesses dos próprios alunos. Acreditamos que é dessa dialética que alunos, professores e gestores dos cursos poderão (re)conhecer o pragmatismo da vida real sem perder de vista as justificativas, interesses e desejos que extrapolam a mera resposta a problemas individuais ou a nichos específicos de mercado de trabalho. Nesse sentido, concordamos que o ensino superior precisa romper com a hegemonia da teoria do capital humano para guiar-se efetivamente pela teoria da emancipação huma$\mathrm{na}^{25}$. Esse é um dos desafios a serem enfrentados para que os caminhos dos CGSC focalizem de fato o perfil de egresso delineado e se direcionem firmemente para os rumos projetados pela Reforma Sanitária Brasileira e para o SUS, fortalecendo-o como uma política de bem-estar social, muito mais do que um mero conjunto de programas e serviços de saúde.

\section{Considerações finais}

Pretendemos identificar, nesta investigação, características sociodemográficas, expectativas e motivações do aluno ingressante dos CGSC, fornecendo com isso subsídios iniciais para a reflexão sobre alguns dos desafios enfrentados no processo de implementação desses cursos.

Constituem limites deste estudo o fato de enfocar apenas um momento inicial dos cursos e não ter envolvido todos os cursos e alunos existentes no momento de sua realização. Porém, foram produzidas informações e levantadas questões pertinentes para estudos comparativos semelhantes, a serem realizados em outros momentos do processo de implementação dos CGSC; bem como, para a realização de estudos qualitativos que venham a aprofundar a compreensão sobre as questões levantadas e até mesmo estudos dos egressos destes cursos.

Entendemos que a escolha de um curso é também a escolha de uma profissão, da entrada ou permanência dos sujeitos no mundo do trabalho e, mesmo, de um projeto de vida que procura responder às inúmeras necessidades pessoais $\mathrm{e}$ familiares, em que pesam visões de mundo e de sociedade, modos de viver, concepções sobre as diferentes áreas e formações profissionais. Nesse sentido, essa escolha pode estar relacionada às diferentes motivações, determinações e construções de identidades. Daí que, neste estudo, não pretendemos exaurir essas motivações, nem explorar suas determinações, mas sim traçar um quadro inicial, exploratório e panorâmico da situação atual. Esperamos que seus resultados e reflexões possam subsidiar o atual processo de implementação dos CGSC, ao identificar questões e desafios a serem enfrentados a curto e médio prazo. Assim, se, por um lado, pretendemos contribuir para o debate geral no campo da Saúde Coletiva; por outro, pretendemos colaborar para a formação de uma base de estudos que viabilizem o acompanhamento e a consolidação da implementação dos CGSC no país. 


\section{Colaboradores}

MEP Castellanos, TLQ Fagundes, TCM Nunes, CRR Gil, ICM Pinto, AS Belisário, SV Viana e RAT Aguiar participaram igualmente de todas as etapas de elaboração do artigo.

\section{Referências}

1. Bosi MLM, Paim IS. Graduação em Saúde Coletiva: limites e possibilidades como estratégia de for mação. Cien Saude Colet 2010; 15(4):2029-2038.

2. Brasil. Decreto $\mathrm{n}^{\circ}$ 6.096, de 24 de abril de 2007 . Institui o Programa de Apoio a Planos de Reestruturação e Expansão das Universidades Federais REUNI. Diário Oficial da União 2007; 25 abr.

3. Gebo KA, Goodyear JD, David SR, Yager JD. Public health studies as an undergraduate major. Public Health Rep 2008; 123(6):812-817.

4. Lee JM. Articulation of undergraduate and graduate education in public health. Public Health Reports 2008; 128(Supl. 2):12-17.

5. Bosi MLM, Paim JS. Graduação em Saúde Coletiva: subsídios para um debate necessário [editorial]. Cad Saude Publica 2009; 25(2):236-237.

6. Teixeira CF. Graduação em Saúde Coletiva: antecipando a formação do sanitarista. Interface Comun Saúde Educ 2003; 7(13):163-166.

7. Paim JS. O objeto e a prática da Saúde Coletiva: o campo demanda um novo profissional. Desafios para a Saúde Coletiva no século XXI. Salvador: EDUFBA; 2006

8. Negri B, Viana ALD, organizadores. O Sistema Único de Saúde em dez anos de desafios. São Paulo: Sobravime, Cealag; 2002

9. Teixeira CF. Formação de recursos humanos para o SUS. Desafios na perspectiva da mudança do modelo de gestão e atenção à saúde. Saúde em Debate 1993; (41):20-23.

10. Gil CRR. Formação de recursos humanos em Saúde da Família: paradoxos e perspectivas. Cad Saude Publica 2005; 21(2):490-498.

11. Oguisso T, Lira PS, Vieira APM, Pereira KCM, Mesquita MMC, Silva PJP. Perfil do Estudante Ingressante no Curso de Graduação da Escola de Enfermagem da Universidade de São Paulo. Rev Paulista Enferm 2006; 25(2):109-116.

12. Spindola T, Martins ERC, Francisco MTR. Enfermagem como opção: perfil de graduandos de duas instituições de ensino. Rev Bras Enfer 2008; 61(2): 164-169.

13. Bardagi MP, Lassance MCP, Paradiso AC. Trajetória Acadêmica e Satisfação com a Escolha Profissional de Universitários em Meio de Curso. Rev. bras. orientac. prof 2003; 4(1/2):153-166.

14. Pierantoni CR. Alunos de graduação em enfermagem - perfil, expectativas e perspectivas profissionais [relatório]. Rio de Janeiro: Instituto de Medicina Social, Universidade do Estado do Rio de Janeiro. Rede Observatório de Recursos Humanos em Saúde, PAHO, WHO Brasil; 2008.
15. Costa AMD, Costa JRV, Costa MD, Costa RD, Botre TEA. Contribuição do Perfil do Aluno de Odontologia para Redefinição dos Recursos Usados pelo Professor no Processo Ensino-Aprendizagem. Rev Fac Odontologia de Lins 2002; 4(1):30-34.

16. Soares DHP. A escolha profissional: do jovem ao adulto. São Paulo: Summus; 2002.

17. Bardagi M, Lassance MCP, Paradiso AC, Menezes IA. Escolha profissional e inserção no mercado de trabalho: percepções de estudantes formandos. Psicol. esc. educ. 2006; 10(1):69-82.

18. Varella TC, Pierantoni CR. Mercado de trabalho: revendo conceitos e aproximando o campo da saúde. A década de 90 em destaque. Physis 2008; 18(3):521-544.

19. Brasil. Ministério da Saúde (MS). Secretaria de Políticas de Saúde. Coordenação Geral da Política de Recursos Humanos. Política de Recursos Humanos para o SUS: balanço e perspectivas. Brasília: MS 2003.

20. Brasil. Ministério da Saúde (MS). Coordenação Geral de Desenvolvimento de Recursos Humanos para o SUS. Regulação e Gestão de Recursos Huma nos em Saúde na Perspectiva da Reforma do Estado. Brasília: MS; 1998.

21. Alves M, Alves N, Chaves M. Inserção profissional e razões de ingresso e reingresso no ensino superior um ponto de partida para uma temática em aberto. Sociologia, Problemas e Práticas 2012; 69:99-118.

22. Ojeda BS, Creutzberg M, Feoli AMP, Melo DS, Corbellini VL. Acadêmicos de enfermagem, nutrição e fisioterapia: a escolha profissional. Rev Lat Am Enfermagem 2009; 17(3):396-402.

23. Spindola T, Martins ERC, Francisco MTR. Enfermagem como opção: perfil de graduandos de duas instituições de ensino. Rev. bras. enferm 2008: 61(2):164-169.

24. Haddad AE, Pierantoni CR, Ristoff D, Xavier IM, Giolo J, Sival LB. A trajetória dos cursos de graduação na área da saúde: 1991-2004. Brasília:Instituto Nacional de Estudos e Pesquisas Educacionais Anísio Teixeira; 2006.

25. Severino AJ. O ensino superior brasileiro: novas configurações e velhos desafios. Educar 2008; 31:7389.

Artigo apresentado em 24/11/2012

Aprovado em 23/02/2013

Versão final apresentada em 12/03/2013 